\title{
Escritura y autoejemplaridad en la Epistula 30 de Séneca
}

\author{
Soledad Correa \\ Universidad de Buenos Aires-CONICET \\ soledad.correa@yahoo.com.ar \\ ORCID iD: http://orcid.org/0000-0002-5367-117X \\ Writing and self-exemplarity in Seneca's Epistula 30
}

La cuestión de la autoejemplaridad senecana en las Epistulae ha sido poco estudiada. Este artículo parte del supuesto de que en ellas Séneca se apropia activamente de un dispositivo cultural clave para la construcción de la memoria en Roma: el discurso ejemplar. Habida cuenta de que aprender a morir bien es uno de los ejes vertebradores del epistolario, examinaremos la Ep. 30, en la que el ego epistolar ofrece una descripción de cómo un amigo, ya anciano, enfrenta de manera ejemplar su trance final. Atendiendo a que debe haber no sólo un registro que visibilice la acción que se propone como ejemplar (Roller 2004), sino también un agente que se proponga realizar dicho registro, intentaremos demostrar que en esta carta el ego epistolar, con el concurso de la escritura, dirige la atención del lector hacia su propia ejemplaridad, con vistas a forjar un retrato positivo y memorable de sí mismo.

Palabras clave: Séneca; Epistulae; autoejemplaridad.
Seneca's self-exemplarity in the Epistulae deserves further attention. This article assumes that in this text Seneca actively appropriates one Roman cultural device which plays a central role in memory building, the discourse of exemplarity. Taking into account that the topic of dying well is one of the backbones of the collection as a whole, we will examine Ep. 30, where the epistolary ego describes how an old friend faces death in an exemplary way. Considering that a deed proposed as exemplar requires not only a record, but also an agent that makes it visible (Roller 2004), we will try to show that in this letter the epistolary ego, aided by the written word, directs the reader's attention to his own exemplarity, in order to build a positive and memorable portrait of himself.

Key words: Seneca; Epistulae; self-exemplarity.

Cómo citar este artículo / Citation: Correa, Soledad 2017: «Escritura y autoejemplaridad en la Epistula 30 de Séneca», Emerita 85 (1), pp. 95-115. 


\section{INTRODUCCIÓN}

Sabemos que Séneca (ca. 5 a. C. - 65 d. C.) compuso sus Epistulae Mora$l e s^{1}$ al final de su vida, en algún momento entre su retiro de la vida pública como amicus principis en el 62 d. C. y su muerte en el 65 d. C. ${ }^{2}$ Probablemente son su última obra y han sido consideradas como la culminación de su labor filosófica, un intento de sintetizar y transmitir la doctrina estoica sobre cómo vivir bien ${ }^{3}$, doctrina que se encuentra esbozada de manera más difusa en el resto de sus escritos filosóficos, y tratada menos directamente en sus obras dramáticas. En comparación con la correspondencia ciceroniana -uno de sus principales modelos literarios-, el corpus epistolar de Séneca es limitado, tanto en su cronología como en cantidad ${ }^{4}$ : ciento veinticuatro cartas en veinte libros han llegado hasta nosotros, todas escritas por Séneca y dirigidas a Lucilio, un miembro del orden ecuestre, procurador de Sicilia, a quien Séneca dedicó también otras dos obras, Naturales Quaestiones y De prouidentia. La tendencia a considerar las Epistulae Morales como ensayos ${ }^{5}$ con fórmulas epistolares adosadas al comienzo y al final de cada carta ha comenzado a revertirse en los últimos años y varios estudiosos han dedicado sus esfuerzos a poner de relieve la fundamental inscripción de la colección en el género epistolar ${ }^{6}$. Aunque la cuestión de si las Epistulae constituyen un genuino intercambio de correspondencia entre Séneca y su destinatario es aún

1 Aulo Gelio es el primero en utilizar este título para referirse a las cartas de Séneca (praef. IX). Es posible, sin embargo, que el título haya sido empleado por el propio Séneca (Cugusi 1983, p. 196).

${ }_{2}$ Para las fechas de composición y publicación, cf. Griffin 1992, pp. 305, 348-350, 400 y 418.

3 Para el lugar de las Epistulae en la obra de Séneca y sus planes de escribir una moralis philosophia adicional, de mayor alcance, cf. Leeman 1953 y Russell 1974. Sobre la presencia de la filosofía en la obra dramática de Séneca, Marti 1945 postuló que las tragedias eran tratamientos sistemáticos de filosofía estoica. A propósito de esta cuestión, cf. Pratt 1948; Leeman 1966; Pociña Pérez 1976; 1978; Brown 1992.

${ }^{4}$ Cf. Wilson 2001, p. 186, quien conjetura que posiblemente la «publicación» de las cartas de Cicerón a Ático en época neroniana inspiró a Séneca a componer una colección epistolar dirigida a un único destinatario. Las cartas de Cicerón a Ático son más de 400 y abarcan un período de veinticinco años (68-43 a. C.).

${ }^{5}$ Cf. Wilson 2001, pp. 165-169, a propósito de lo que él denomina «lecturas ensayísticas» de las cartas.

${ }^{6}$ Cf., p. ej., Cancik 1967; Maurach 1970; Wilson 1987; 2001; Mazzoli 1989. 
objeto de debate 7 , no resulta plausible pensar que fueron escritas solo para Lucilio, dada su abarcadora agenda filosófica y su aspiración a alcanzar un público más amplio, e incluso a la posteridad, a quien Séneca dice dirigirse especialmente ${ }^{8}$.

Uno de los problemas a los que debe hacer frente el estudioso de Séneca es el hecho de que, a pesar de que éste escribió voluminosamente y en primera persona, lo que sabemos sobre su vida es muy poco ${ }^{9}$. En efecto, si hay una característica que diferencia al epistolario senecano del de Cicerón (uno de sus principales modelos genéricos, según ya señalamos) es que las cartas de Séneca guardan un elocuente silencio respecto de sus condiciones socio-históricas de producción y circulación, pues nada mencionan sobre Nerón, ni sobre el papel que Séneca desempeñara primero como magister o praeceptor y luego como amicus principis. Las Epistulae son, con todo, la obra más rica en detalles personales de todo el corpus senecano, pues una exigencia básica del género epistolar es revelar los rasgos de carácter del escritor ${ }^{10}$. Sin embargo, en la colección epistolar senecana lo biográfico -sea que se trate de la propia vida del escritor o de sucesos de la vida del destinatario o de otros amici ${ }^{11}$ - sólo irrumpe a condición de que sea ejemplar ${ }^{12}$. Como es sabido, el proyecto de la obra como un todo es instar a Lucilio a emprender un serio examen de sí mismo y un distanciamiento reflexivo de la vida política. Examinarse a sí mismo

7 Para una discusión sobre la historia de las opiniones sobre la «autenticidad» de las cartas, cf. Mazzoli 1989 y Graver 1996, pp. 13-24.

${ }^{8}$ Cf. Sen., Ep. 8.2. Cf., asimismo, Ep. 21.5, 22.2, 64.7 y 79.17.

${ }^{9}$ Cf. Syme 1958, p. 552, «Without the testimony of Tacitus, Seneca the statesman could hardly exist».

${ }^{10}$ Cf. Muñoz Martín 1985, p. 88. En el De elocutione (§227), obra atribuida erróneamente a Demetrio de Falero, se menciona también esta característica de la carta. Sobre este tratado, cf. Malherbe 1988.

${ }^{11}$ Para hechos de la vida de Lucilio, cf., p. ej., Sen., Ep. 28 y 47; para episodios de la vida de otros amici, cf., p. ej., Ep. 27.5; 30; 55.2-7; 101.

${ }_{12}$ Como apunta Griffin 1992, p. 5, «... he is more concerned to offer the public examples of the moral preacher, the pedagogue, the struggling student, the zealous convert, than to portray his real relationship with his addressee Lucilius, or record his own moods». De acuerdo con esto, puede pensarse que las narrationes ejemplares que Séneca utiliza como disparadoras de la reflexión filosófica tienen un carácter doblemente marcado, no sólo porque son escasas, sino también porque suelen colocarse en el incipit de la carta: cf. Sen., Ep. 7.34 ; 12.1-3; 18.1-3; 26.1-3; 30; 49.1; 50.2-3; 51.1; 53.1-4; 54; 55.1-4; 56.1-2; 57.1-3; 64.1-2; $65.1-2 ; 66.1-4 ; 67.1-2 ; 70.1 ; 76.1-4 ; 77.1-9 ; 80.1-2 ; 83.1-7 ; 86.14-21 ; 87.1-5 ; 91.1-3 ; 101.1-4$; $104.1-5 ; 108.13-23 ; 123.1-2$. 
en términos de fortalezas y debilidades es una condición sine qua non para vivir bien realizando progresos hacia el ideal estoico de la perfección racional o bona mens. A lo largo de las Epistulae se insiste en el hecho de que este autoexamen no puede ser llevado a cabo por el proficiens en forma solitaria o sin una guía externa ${ }^{13}$. Los modelos son necesarios, sea bajo la forma del $s a$ piens estoico o de paradigmas históricos como Sócrates o Catón, en la medida en que estas figuras ejemplares proveen al ego actual (occurrent self) de un estándar o «identidad normativa» con la cual llevar a cabo el autoexamen (Long 2009, p. 26). De acuerdo con esto, varios trabajos insisten en señalar el lugar central que el discurso ejemplar en tanto práctica cultural ocupa en la obra de Séneca, en general, y en las Epistulae, en particular ${ }^{14}$. Así, Nussbaum 1994, p. 430, ha caracterizado la colección epistolar senecana como «one long rich exemplum». Por su parte, Mayer 2008 analiza la funcionalidad de los exempla históricos en toda la obra de Séneca e indica que, a diferencia de lo que ocurre en las tragedias y en sus otras obras en prosa, en las Epistulae, a excepción de las Ep. 24 y 71, Séneca hace poco uso de uno de sus recursos literarios predilectos, esto es, la agrupación de exempla en listas ${ }^{15}$. Por su parte, Inwood 2009 sostiene que la novedad de las Epistulae -en las que Séneca da a la filosofía estoica una forma que no se deriva automáticamente de sus modelos griegosreside en el hecho de que en ellas el ego epistolar parece tener una particular predilección por citar su propia experiencia como exemplum, cuestión que ya había sido advertida por Edwards 1997, p. 23. Sin embargo, Inwood no problematiza estas incursiones del ego epistolar en el ámbito de la ejemplaridad por considerarlas como una «natural» consecuencia del género ${ }^{16}$. Asimismo, Roller

13 En lo que atañe a la necesidad de contar con jueces internos y externos de las propias acciones, cf. Bartsch 2006, pp. 191-208 y Roller 2001, pp. 84-88.

14 En la cultura romana, la ejemplaridad es un discurso, un sistema coherente de símbolos que organiza el pasado de una manera particular, determina una manera también particular de fijarlo, conocerlo y emplearlo. A propósito de la estructura y rasgos del discurso ejemplar, cf. Roller 2004, pp. 1-7 y Schiesaro 2009. Para enfoques específicamente historiográficos de esta cuestión, cf. Chaplin 2000 y Walter 2004, pp. 51-70.

15 Para este tema, cf. Roller 2015. Para explicar el hecho de que Séneca haga poco uso de exempla en las Epistulae, Mayer 2008, pp. 307-308, ofrece dos razones: «First, the use of exempla, just because it was inculcated as a rhetorical ornament of literary style, must have seemed alien to the familiar style appropriate to a personal letter...Secondly, where illustration was wanted, the immediate experience of the correspondents was likely to provide it».

${ }^{16} \mathrm{Cf}$. Inwood 2009, p. 58, cursivas en el original: «It is Seneca’s decision as a writer to make this insertion that should draw our attention. It is both a deliberate and controlled act 
2016 muestra que, si bien Séneca explota sistemáticamente esta práctica cultural distintivamente romana, esto es, el uso de exempla como modelos de acción en el presente, las Ep. 94 y 108 someten este discurso a un examen crítico que arroja luz sobre sus deficiencias, aunque en ningún momento se sugiere abandonarlo.

Este breve estado de la cuestión tiene como propósito sugerir que la idea de que el ego de las Epistulae se construye a sí mismo como exemplum -aunque ha sido insinuada por algunos estudiosos- parece requerir un examen más detallado. Antes de proponer nuestras hipótesis para la Ep. 30, parece oportuno ofrecer aquí un rápido repaso de las instancias en las que el texto hace mención explícita de la necesidad de que el proficiens elija a un otro como figura idealizada, conforme a la cual modelar la propia vida: en la $E p$. 11.8-10 el ego epistolar señala que buena parte de las faltas (peccatorum) pueden evitarse si uno se imagina bajo la mirada de un custos o paedagogus. Puede tratarse de paradigmas históricos como Catón o Lelio o «de alguien cuya conducta, palabras y semblante, espejo del alma» (et uita et oratio et ipse animum ante se ferens uultus) «te resulten agradables» (tibi placuit). Más adelante, en la $E p$. 29.6, se señala que, «una vez que haya progresado hasta el punto de tener respeto incluso de sí mismo» (cum iam profeceris tantum ut sit tibi etiam tui reuerentia), Lucilio «podrá despedir al preceptor» (licebit dimittas paedagogum). Mientras tanto, habrá de protegerse con el ascendiente (auctoritas) que ejerza alguien sobre él, ya sea Catón, Escipión, Lelio o «cualquier otro cuya sola presencia lograría suprimir los vicios de los depravados» (alius cuius interuentu perditi quoque homines uitia supprimerent). En la Ep. 32.1, el ego asume directamente el papel de paedagogus: «vive como si yo fuera a oír qué haces, más aún, como si yo fuera a verlo» (sic uiue tamquam quid facias auditurus sim, immo tamquam uisurus). Finalmente, en la Ep. 52.7 se reitera la idea de que, dado que el camino hacia la sabiduría está plagado de obstáculos (imus per obstantia), el proficiens ha de buscar ayuda no sólo entre los presentes, sino también entre los antiguos (adiunare nos possunt non tantum qui sunt, sed qui fuerunt). La clave para

by an immensely artful writer and a natural and unproblematic feature of the genre of letter writing. What could be more natural than that I talk about myself when I write a letter to a friend? What could be more natural than that I should reveal a personal insight or vulnerability? Or that I should wield a pointed tu quoque argument with someone who I know shares my basic values and inclinations? Having decided to write philosophical letters Seneca can and indeed should intrude himself into the argument often an effectively». 
elegir a alguien entre los presentes será optar por aquel a quien se admira más por haberle visto que por haberlo escuchado (eum elige adiutorem quem magis admireris cum uideris quam cum audieris, 52.8). Aunque Bartsch señala que los exempla que Séneca nos propone son siempre personajes idealizados del pasado ${ }^{17}$, consideramos que, en las Epistulae, el ego epistolar construye su ethos discursivo bajo el signo de la ejemplaridad, apropiándose activamente de este tipo de discurso ${ }^{18}$, cuestión que ilustraremos a partir de un análisis de la Ep. 30.

Dado que en las cartas se reitera la idea de que la proximidad de la muerte ofrece una prueba definitiva del carácter de un individuo ${ }^{19}$, analizaremos la Ep. 30, en la que el ego epistolar ofrece una descripción de cómo un amigo, ya anciano, Aufidio Baso, enfrenta de manera ejemplar su trance final. Por lo demás, la idea de la muerte como un espectáculo edificante es conspicua en varios textos latinos, donde morir es configurado como un proceso activo, que constituye un acto social de comunicación con los vivos caracterizado por su capacidad para revelar el verdadero carácter de quien muere

${ }^{17}$ Por consiguiente, considera que el pasaje antes citado (Sen., Ep. 32.1), en el que se insta al destinatario a imaginarse bajo la mirada del ego epistolar, «... is a rare case of a contemporary witness as imaginary other. Although Seneca tells Lucilius that we can select our guides from both the living and the dead..., no such living exemplum is offered as a possibility» (Bartsch 2006, p. 202). Cf., sin embargo, Griffin 1992, p. 4: «Of the living, Seneca himself is the main exemplum».

${ }^{18}$ Hay dos momentos en el epistolario que, a nuestro juicio, permiten considerar seriamente esta posibilidad: en la Ep. 24 el ego epistolar induce al destinatario a minimizar su preocupación ante el resultado de un proceso reuniendo exempla que lo reconforten: Metelo, Rutilio, Sócrates, Mucio Escévola son mencionados, hasta que Lucilio lo interrumpe con una importante objeción (24.6): "“son éstas", alegas, "historias (fabulae) repetidas a coro (decantatae) en todas las escuelas; en el momento en que pasemos a tratar del desprecio de la muerte me contarás la suerte de Catón"». Es interesante que estos relatos ejemplares sean designados como fabulae, pues si tomamos este sustantivo en su acepción dramática (cf. OLD, s. u. 6: «a play, drama») puede pensarse que lo que se cuestiona aquí no es el discurso ejemplar per se, sino su lectura o recepción, es decir, el hecho de que el receptor de exempla se vea reducido a un papel de espectador pasivo. No es casual, entonces, que el ego epistolar ponga esta objeción en boca de Lucilio, emblema del lector externo, ya que las Epistulae apuntan a construir un modelo de lector activo (cf. Sen., Ep. 33, 79 y 84). Esto se ve corroborado más adelante en la Ep. 98.13: «hagamos también nosotros alguna gesta valerosa: situémonos entre los modelos» (nos quoque aliquid et ipsi faciamus animose; simus inter exempla).

19 Cf., p. ej., Sen., Ep. 26.6: mors de te pronuntiatura est. 
(Edwards 2007, p. 5) ${ }^{20}$. Si consideramos las Epistulae de Séneca en su conjunto puede afirmarse que aprender a morir bien (bene morior, Ep. 61.2) es uno de los ejes vertebradores del epistolario. En efecto, el hilo conductor de estas 124 cartas de variada extensión dirigidas a Lucilio es la recurrencia de determinadas preocupaciones e imágenes, entre las cuales la muerte figura de manera prominente ${ }^{21}$. Dado que sabemos que el epistolario es una obra estructurada a modo de «red entrelazada» (Cancik 1967, Maurach 1970) y, por lo tanto, el orden en que están dispuestas las cartas es significativo, conviene detenerse brevemente en el contexto más inmediato de la $E p$. 30: está precedida por la Ep. 29, que es la última en ofrecer una cita de Epicuro, Metrodoro, o de algún otro seguidor de Epicuro como forma de cancelación de una pretendida deuda con Lucilio, e inaugura una breve serie de cartas que omiten el pago de pequeños regalos (munuscula, Ep. 10.5) o deudas sin comentario alguno. Esta breve serie termina cuando el ego epistolar se rehúsa a acceder al pedido de Lucilio de reanudar esta costumbre al comienzo de la $E p .33^{22}$. Si recordamos que en la Ep. 6.5 se señala que el camino (iter) hacia la sabiduría es largo (longum) a través de los preceptos (per praecepta), pero breve y eficaz (breue et efficax) a través de ejemplos (per exempla), puede considerarse que la $E p .30$ marca el comienzo de una nueva dinámica epistolar que concederá un mayor énfasis al discurso ejemplar ${ }^{23}$. De esta manera, Baso es

${ }^{20}$ «Dying, for Roman writers, is...not a private act but one which should properly take place in front of an audience» (Edwards 2007, p. 144).

${ }^{21}$ Cf., p. ej., Sen., Ep. 1.2; 4.3; 12.6; 26.6 y 9; 77.6. A propósito de la centralidad del tema de la muerte en la obra de Séneca, cf. Ker 2009.

${ }^{22}$ Es interesante señalar que, si bien en esta dinámica de sustitución de praecepta por exempla que se inicia luego de la Ep. 29 el contenido doctrinal del epicureísmo se amplía, en la Ep. 30 el mensaje de Epicuro está puesto en boca de un sujeto moribundo. Cf. Wildberger 2014, p. 450: «One might even read Ep. 30 as a symbol of Seneca's departure from his previous alignment with Epicurus, which is laid to rest, so to speak, with the dying Epicurean Bassus».

${ }^{23}$ Cf. Wilcox 2012, p. 134: "The new emphasis on exemplary discourse that the appearance of Aufidius Bassus heralds does not replace epistolarity as a mode of writing or correspondence as a medium for friendship. Instead, the exemplary discourse of letter 30 offers a complementary model for both reading and living». Asimismo, consideramos que este nuevo énfasis en el discurso ejemplar puede considerarse como un intento de darle a su proyecto filosófico por vía epistolar una impronta netamente romana. En efecto, Cicerón señala que Roma ha superado a Grecia en la provisión de exempla de moderación (Fin. II 62), y que la Vrbs está repleta de ellos (Off. III 47). Más tarde, Quintiliano se hará eco de esto e indicará que mientras que los griegos se destacan por sus praecepta, los romanos los aventajan en el 
el primer exemplum viviente explícitamente promovido por el texto de las Epistulae, a punto tal que una carta entera se le dedica al spectaculum de su ejemplaridad ${ }^{24}$.

Ahora bien, a pesar de que en la Ep. 30 se promueve explícitamente el exemplum de Baso, según veremos, el ego epistolar también se configurará como un modelo de alguien que está aprendiendo a morir bien, aunque su ejemplaridad operará de un modo diferente a la de Baso: mientras que Baso aprende de manera directa, por su propia experiencia, sobrellevando el debilitamiento extremo de su cuerpo y glosando este proceso y su significado, el remitente se presentará en proceso de aprendizaje de la misma lección (cómo morir bien) a la distancia, observando y haciendo un registro textual sobre la experiencia de Baso y su propia reacción ante ella. Atendiendo a que debe haber no sólo un registro, un monumentum, que visibilice la acción que se propone como ejemplar (Roller 2004), sino también un agente que se proponga realizar dicho registro puede considerarse que el ego epistolar, con el concurso de la escritura ${ }^{25}$, logrará con esta carta dirigir la atención del lector hacia su propia ejemplaridad, con vistas a forjar un retrato positivo y memorable de sí mismo.

\section{LA MUERTE de BASO EN LA EP. 30: entre EL SPECTACULUM Y EL EXEMPLUM}

La $E p .30$ se abre sin preámbulos epistolares o referencias al destinatario, y va directamente al grano: el primer párrafo es una vívida introducción no sólo de Baso sino también del libro cuarto de las Epistulae: Bassum Aufidium, uirum optimum, uidi quassum, aetati obluctantem ${ }^{26}$. Como puede apreciarse,

establecimiento de exempla (XII 2.30). Este aparente triunfo de las acciones sobre los textos no implica, sin embargo, que sea posible prescindir de los textos para dar perdurabilidad a las acciones.

${ }^{24}$ Es interesante el modo paradójico en que en la figura de Baso confluyen praecepta y exempla. En efecto, en 30.14 el ego epistolar presenta a Baso, un exemplum explícitamente promovido a lo largo de la Ep. 30, siguiendo los praecepta de Epicuro.

${ }^{25}$ Es preciso señalar que este interés por registrar el modo en que un individuo enfrenta la muerte de ningún modo es exclusivo de Séneca, sino que forma parte de un fenómeno de mayor alcance: «...Rome in the first and early second centuries CE was characterised by a vogue for death literature. The endless retellings of Cato's death are a symptom of this which also served to fuel the appetite for details of the deaths of other great Romans» (Edwards 2007, p. 131).

${ }^{26}$ Sen., Ep. 30.1. Seguimos la edición de Reynolds 1965. 
Baso es presentado como uir, término ligado etimológicamente a uirtus ${ }^{27}$, que posee connotaciones militares. La uirtus de Baso, con todo, es de una índole especial, pues no se dirime en el campo de batalla sino en una lucha abstracta y desigual contra el deterioro de su propio cuerpo ${ }^{28}$. Por otra parte, esta imagen de Baso en combate evoca un tipo de muerte arquetípica en Roma: la del gladiador en la arena ${ }^{29}$. Asimismo, esta introducción puede considerarse clave por establecer la delicada tensión entre spectaculum y exemplum ${ }^{30}$ que el ego epistolar sostendrá a lo largo de toda la carta $^{31}$.

${ }^{27}$ Cf. Cic., Tusc. II 43: Appellata est enim ex uiro uirtus; uiri autem propria maxime est fortitudo, cuius munera duo sunt maxima, mortis dolorisque contempti.

${ }^{28}$ Cf. Edwards 2007, p. 7: «Despite the militaristic nature of Roman society, death in war...receives only limited attention in Roman discussions of death. Yet, as Roman aristocrats are increasingly excluded from high-profile military campaigns during the principate, the process of dying seems to become a new arena for the exercise of a quasi-martial virtue...». Cf., asimismo, Roller 2001, p. 104: «The proper arena for exercising uirtus is now not warfare, but the endurance of ill fortune». Para la imagen del combate en la obra de Séneca, cf. Armisen-Marchetti 1989, pp. 94-97.

${ }^{29} \mathrm{La}$ imagen del gladiador es empleada más adelante en esta misma carta (§8), como un modelo para hacer frente a la muerte con la disposición mental adecuada, a pesar de la profunda ambivalencia que los escritores romanos miembros de la élite experimentaban hacia ellos (para referencias a la brutalidad de los munera en las Epistulae, cf. Sen., Ep. 7.3-8; 95.33; 103.2). Es posible rastrear elementos de esta identificación con el gladiador en época republicana e incluso antes: «The scrutiny under which the gladiator played his role might in some sense be construed as parallel to the regard which was fixed on the Roman magistrate or general competing for public honours. The gladiator could be seen as a mirror image of the aristocrat in this society of spectacle... Rome's leading citizens, like gladiators, must perform in accordance with the expectations of their audience. Their identity, like that of those same gladiators, is contingent on the recognition afforded them by that audience» (Edwards 2007, pp. 74-75). La fascinación de los miembros de la élite por la figura del gladiador ha sido interpretada como una respuesta a su cambio de posición bajo líderes autocráticos: cf. Barton 1993. Cf., asimismo, Armisen-Marchetti 1989, pp. 124-126.

${ }^{30}$ Cf. Solimano 1991, p. 43: «L'exemplum senecano, vero e proprio sermo corporeus, si configura... non tanto come narrazione, quanto come quadro singolo provocante, come messa in scena di un gesto di grande effetto spettacolare, talvolta accompagnato da una battuta di sapore teatrale».

${ }^{31}$ A propósito de esta tensión, cf., p. ej., Sen., Ep. 78.21: Est, mihi crede, uirtuti etiam in lectulo locus. Non tantum arma et acies dant argumenta alacris animi indomitique terroribus: et in uestimentis uir fortis apparet. Habes quod agas: bene luctare cum morbo. Si nihil te coegerit, si nihil exorauerit, insigne prodis exemplum. O quam magna erat gloriae materia, si spectaremur aegri! ipse te specta, ipse te lauda. Cf., asimismo, Sen., Ep. 66.4, en la que se alude a un amigo, Clarano, como exemplar. 
El párrafo siguiente establece un contraste entre el cuerpo senil de Baso (in senili corpore, §2), cuya debilidad (imbecillitas, §2) es comparada con un navío maltrecho (nauigio dehiscenti, §2) ) $^{32}$ con un edificio carcomido (in putri aedificio, §2), y el estado de su espíritu:

Bassus tamen noster alacer animo est: hoc philosophia praestat, in conspectu mortis hilarem <esse> et in quocumque corporis habitu fortem laetumque nec deficientem quamuis deficiatur. Magnus gubernator et scisso nauigat uelo et, si exarmauit, tamen reliquias nauigii aptat ad cursum. Hoc facit Bassus noster et eo animo uultuque finem suum spectat quo alienum spectare nimis securi putares ${ }^{33}$.

La distancia con que Baso puede considerar su propio fin como si no fuese propio es resultado de la acción liberadora de la filosofía, capaz de eliminar el principal obstáculo que impide a los hombres vivir la clase de vida que la naturaleza quiere para ellos: el miedo a la muerte ${ }^{34}$. De hecho, puede considerarse que aquí se alude a la definición socrática de la filosofía como constante preparación para la muerte, pues la cogitatio mortalitatis o meditatio mortis es identificada por el Sócrates platónico como el acto que define al filósofo ${ }^{35}$. Esta meditatio mortis es explícitamente equiparada con una meditatio libertatis en la Ep. 26, donde se señala que quien aprendió a morir, desaprendió la esclavitud (qui mori didicit seruire dedidicit, §10). Asimismo, vemos aquí que el spectaculum de la propia muerte está puesto en abismo: Baso spectator es contemplado por Séneca, que, a su vez, es contemplado/

${ }^{32}$ El imaginario del viaje por mar, incluyendo sus técnicas y, sobre todo, sus peligros, presenta, junto al del combate y al de la medicina, la coherencia de un esquema, cuyo simbolismo se aplica tanto al plano de la vida ordinaria como al de la vida moral. Cf. Armisen-Marchetti 1989, pp. 140-142.

33 Sen., Ep. 30.3.

${ }^{34}$ Cf. Sen., Ep. 4.3-6 y 8-9. El tratado senecano De tranquillitate animi pone el foco en el miedo a la muerte, considerándolo como una emoción profundamente desestabilizadora, que, en caso de no ser controlada, puede trastocar completamente la vida: male uiuet quisquis nesciet bene mori (11.4).

${ }^{35}$ Cf. Pl., Phd. 81a. Séneca recuerda esto en Marc. 23.2: inde est quod Platon clamat: sapientis animum totum in mortem prominere...Al mismo tiempo, puede considerarse que aquí se evoca también la muerte de Sócrates, que funcionará como una suerte de guion o arquetipo para muertes «filosóficas» subsiguientes, en especial, para el paradigmático suicidio de Catón el Joven, a pesar de que éste, a diferencia de Sócrates, no fue condenado a muerte sino que optó por darse muerte a sí mismo. Para una evocación de la muerte de Catón en las Epistulae, cf. Sen., Ep. 24.6-8. 
leído por Lucilio y por el lector moderno. Este recurso permite trazar un paralelo no sólo entre la mirada y la lectura, implicando al lector en el juego, sino también entre Baso y el ego epistolar, cuya primera presentación en el texto, según vimos, se da a través del verbo uidi que abre la carta. Sin embargo, dado que, siguiendo el modelo del Fedón, en lo sucesivo el foco estará puesto en el discurso de Baso y no en el sufrimiento físico que padece ${ }^{36}$, en adelante el ego epistolar irrumpirá en el texto mediante ocurrencias del verbo audio (audiebam, §9; audio, §15).

El paralelo entre Baso y el ego epistolar se insinúa de hecho desde el inicio mismo de la carta, a partir de la referencia a la debilidad crónica (semper infirmi corporis et exsucti fuisse, §1) y vejez (senectus, §1) que afectan al cuerpo del amigo ${ }^{37}$. Este paralelo se ve reforzado en el párrafo 2, pues la comparación entre el cuerpo senil de Baso y un edificio carcomido establece una relación de intertextualidad interna entre esta carta y la Epistula 12, con la que concluye el libro primero de las Epistulae. En efecto, allí el ego epistolar exhibe su propia senectus, que se le presenta metaforizada bajo la forma de una uilla arruinada por el paso del tiempo ${ }^{38}$. Con todo, es interesante que Séneca no mencione explícitamente su vejez en esta carta, sino que ésta emerja como un efecto de la lectura continua del epistolario. En efecto, en la Ep. 26.1, por ejemplo, había señalado: inter decrepitos me numera et extrema tangentes. Aquí, sin embargo, el ego epistolar rehúsa insertar comentarios sobre su edad avanzada y se refiere a la vejez más como spectator a la distancia que como alguien que padece los achaques que trae aparejados. En la carta que nos ocupa el ego entreteje su descripción del comportamiento y dichos ejemplares de Baso con sus propias meditaciones. De acuerdo con esto, señala que la muerte por vejez se distingue de otros tipos de muerte por

${ }^{36}$ Cf. Gill 1973, quien señala que los síntomas de Sócrates en el Fedón no se condicen con los pronunciados efectos que la cicuta tiene sobre el cuerpo.

${ }^{37}$ El tema de la mala ualetudo de Séneca es puesto en primer plano en la Ep. 54, con la descripción de los ataques de asma que padece, que los médicos llaman «meditatio mortis» (Sen., Ep. 54.2). Según afirma, el asma lo ha entrenado para estar listo para morir: Hoc tibi de me recipe: non trepidabo ad extrema, iam praeparatus sum, nihil cogito de die toto (Ep. 54.7). La misma idea se reitera en la Ep. 70.18: nullius rei meditatio tam necessaria est; ... huius unius rei usum qui exigat dies ueniet.

${ }^{38}$ Cf. Sen., Ep. 12.1: Quocumque me uerti, argumenta senectutis meae uideo. Veneram in suburbanum meum et querebar de impensis aedificii dilabentis. Ait uilicus mihi non esse neglegentiae suae uitium, omnia se facere, sed uillam ueterem esse. Haec uilla inter manus meas creuit: quid mihi futurum est, si tam putria sunt aetatis meae saxa? 
no dar lugar a la esperanza: alia genera mortis spei mixta sunt (Ep. 30.4). Esta cuestión es ilustrada con una congeries: desinit morbus, incendium exstinguitur, ruina quos uidebatur oppressura deposuit; mare quos hauserat eadem ui qua sorbebat eiecit incolumes; gladium miles ab ipsa perituri ceruice reuocauit (Ep. 30.4). Esta acumulación es rematada por una lapidaria conclusión: nil habet quod speret quem senectus ducit ad mortem (Ep. 30.4). Otra singularidad de la muerte por vejez es que ocurre en forma suave y «en cámara lenta»: nullo genere homines mollius moriuntur sed nec diutius (Ep. 30.4). Esta lentitud ${ }^{39}$ habilita acaso la posibilidad de ofrecer no sólo una performance ejemplar de la propia agonía, sino también de convertir esta misma agonía en ocasión para la indagación ética ${ }^{40}$ :

Bassus noster uidebatur mihi prosequi se et componere et uiuere tamquam superstes sibi et sapienter ferre desiderium sui. Nam de morte multa loquitur et id agit sedulo ut nobis persuadeat, si quid incommodi aut metus in hoc negotio est, morientis uitium esse, non mortis; non magis in ipsa quicquam esse molestiae quam post ipsam ${ }^{41}$.

Varios elementos son aquí dignos de nota: en primer lugar, dado que los escritores romanos suelen comparar la vida con una obra o un banquete ${ }^{42}$, es decir, con un proceso estructurado con una conclusión inevitable, el ego epistolar pone el acento en la puesta en escena que Baso hace de su propia muerte (id agit $)^{43}$. Con todo, según podemos advertir, explotando las diferentes valencias de uidere/-eri (verídica o ilusoria) ${ }^{44}$, el simulacro del funeral de Baso

39 Más adelante se señala que morir de viejo requiere una lenta animi firmitas (Ep. 30.8).

${ }^{40}$ Cf. Hill 2004, pp. 178-182.

${ }^{41}$ Sen., Ep. 30.5.

${ }^{42}$ Cf., p. ej., Lucr. III 931-939 y Hor., Serm. I 118-119. Cf. Coffta (2002). La imagen del banquete es evocada más adelante en esta misma carta: Iam uero si cui contigit ut illum senectus leuiter emitteret, non repente auulsum uitae sed minutatim subductum, o ne ille agere gratias diis omnibus debet quod satiatus ad requiem homini necessariam, lasso gratam perductus est (30.12). Para la compleja relación entre la muerte y los conuiuia, cf. Edwards 2007, pp. 161-178.

${ }^{43}$ Cf. OLD ago, s. u. 25 «to stage or act». Cf., asimismo, quomodo fabula, sic uita: non quam diu, sed quam bene acta sit, refert. Nihil ad rem pertinet quo loco desinas. Quocumque uoles desine: tantum bonam clausulam inpone (Sen., Ep. 77.20); magnam rem puta unum hominem agere (Sen., Ep. 120.22).

${ }^{44}$ Para este tema, cf. Reinhardt 2016. 
es presentado no como una realidad sino como producto de la actividad interpretativa de ego epistolar (uidebatur mihi). Esta referencia al aparente funeral de Baso recuerda también otro pasaje de la Epistula 12 antes mencionada, focalizado en la teatralidad literal de los funerales de un cierto Pacuvio (Ep. 12.8), quien ningún día dejó de celebrar su propio entierro. Si bien ensayar mentalmente la propia muerte es un ejercicio recomendable para el proficiens como recordatorio de que la muerte puede llegar en cualquier momento, la necesidad que Pacuvio tiene de repetir a diario este rígido ritual apotropaico configura a este personaje como un exemplum e contrario. En efecto, la Ep. 12 presenta a Pacuvio como alguien que, lejos de haber aprendido cómo morir bien, reconciliándose así con su propia mortalidad, es un esclavo del miedo a la muerte ${ }^{45}$. La intertextualidad interna con la Epistula 12 contribuye así a reforzar la ejemplaridad de la actitud asumida por Baso ante su próxima muerte, spectaculum que nada tiene que ver con despliegues altisonantes.

Otra cuestión destacable en este pasaje es el hecho de que el ego epistolar se configura como una pieza fundamental para el registro y recreación de la performance discursiva (de morte multa loquitur) que Baso hace de su propia muerte ${ }^{46}$. Así, el término multa sugiere que lo que se consigna por escrito es necesariamente una síntesis de un discurso más extenso, en el que se advierten ecos lucrecianos ${ }^{47}$, a pesar de que la filiación epicúrea de Baso sólo se

45 «Pacuvius is locked inside the circle that is his life, consigned to endless, tedious repetition of the same action» (Habinek 1982, p. 69). Cf., asimismo, Mann 2006.

${ }^{46}$ El ego como spectator/auditor se refiere dos veces al placer que le reporta este spectaculum: libentissime itaque illum audiebam quasi ferentem de morte (Ep. 30.9); libenter haec, mi Lucili, audio... (Ep. 30.15).

${ }^{47}$ Como es sabido, en De rerum natura (escrito probablemente a principios de los años 50 a. C.) Lucrecio se propone ofrecer una exposición de la filosofía de Epicuro en hexámetros latinos. Según el poeta, la filosofía de Epicuro ofrece una cura para uno de los mayores males que padece la humanidad, el miedo a la muerte. El libro tercero se concentra específicamente en este tema, que es abordado a partir de un examen minucioso de la estructura del cuerpo humano. Cf., en este sentido, Sen., Ep. 30.11, pasaje que recuerda la prosopopeya de la natura en el texto de Lucrecio (III 931-975), uno de los más conocidos del poema, destacable por el empleo de metáforas derivadas de la ley y por la abundancia de términos arcaicos y neologismos: mors necessitatem habet aequam et inuictam: quis queri potest in ea condicione se esse in qua nemo non est? prima autem pars est aequitatis aequalitas. Sed nunc superuacuum est naturae causam agere, quae non aliam uoluit legem nostram esse quam suam: quid quid composuit resoluit, et quidquid resoluit componit iterum. A propósito de Séneca y Lucrecio, cf. Schottlaender 1955, pp. 141-142, Mazzoli 1970, pp. 206-209; Lana 1991, pp. 263-268; Setaioli 1991; La Penna 1994; Schiesaro 2015, p. 239. 
explicita más adelante en esta misma carta ${ }^{48}$. Esta demora del texto en desvelar el hecho de que Baso es seguidor de las enseñanzas de Epicuro puede explicarse como una tentativa de focalizar la atención del lector en lo dicho y no en la identidad filosófica del emisor, preocupación que también remite al cierre de la Epistula 12, donde se señala que las mejores cosas, sea quien fuere que las haya enunciado, son «patrimonio común» (communia $)^{49}$. Hay, además, otro sentido en que el discurso de Baso es communis $^{50} \mathrm{y}$, así, dos veces en la Epistula 30 el ego epistolar pone de relieve el hecho de que el contenido del discurso de Baso no presenta nada nuevo ${ }^{51}$, anticipándose tal vez a que el lector podría considerarlo remanido. La identidad verbal entre el discurso de Baso y otros discursos sobre la muerte, sin embargo, no constituye un menoscabo de su auctoritas, en la medida en que Baso habla de una muerte próxima. Puede pensarse que la auctoritas de Baso y su ejemplaridad se proyectan no sólo hacia la Epistula 30, único registro de su discurso, sino también hacia el ego epistolar ${ }^{52}$. En este sentido, vale la pena detenerse en el párrafo 7 , en el que se establece una clara jerarquía:

Haec ego scio et saepe dicta et saepe dicenda, sed neque cum legerem aeque mihi profuerunt neque cum audirem iis dicentibus qui negabant timenda a

${ }^{48}$ Cf. dicebat quidem ille Epicuri praeceptis obsequens... (Ep. 30.14). Existe una abundante bibliografía secundaria sobre la presencia de Epicuro en las Epistulae de Séneca. Para una síntesis de los estudios sobre este tema, cf. Graver 2016, p. 192, n. 4.

${ }^{49}$ Cf. Sen., Ep. 12.11: 'Epicurus' inquis 'dixit: quid tibi cum alieno?' Quod uerum est meum est; perseuerabo Epicurum tibi ingerere, ut isti qui in uerba iurant nec quid dicatur aestimant, sed a quo, sciant quae optima sunt esse communia.

${ }^{50}$ Cf. OLD communis, -e, s. u. 7 «common, ordinary».

${ }^{51}$ Cf.. Haec ego scio et saepe dicta et saepe dicenda (Ep. 30.7) y libenter haec, mi Lucili, audio non tamquam noua (Ep. 30.15).

52 Como apunta Schiesaro 2009, p. 55 (cursivas en el original): «Seneca is the chief exponent of a deep revision of the Republican exempla tradition, one in which even the ancient exemplum is suspiciously scrutinized, and there turns out to be never enough evidence of a positive exemplarity. This is because recorded actions are no longer a reliable guide to the intentions and moral dispositions that produce them. That is, what if those people, back then, had been performing exemplary actions for reasons that remain hidden and do not pass the test of moral examination? Needless to say this revision valorizes the role of the 'literary' author over that of the 'author of exempla', and it displaces moral authority from the exemplary hero onto Seneca himself, while pushing past traditions dangerously close to the area of 'display of virtue' instead of 'virtuous action'». 
quorum metu aberant: hic uero plurimum apud me auctoritatis habuit, cum loqueretur de morte uicina.

En efecto, aunque es posible leer y escuchar discursos sobre la muerte, el de Baso tiene la mayor auctoritas, en la medida en que habla de su muerte próxima. Por otra parte, aunque de acuerdo con esta jerarquía, leer un texto sobre un comportamiento ejemplar no puede tener la misma auctoritas que contemplar el exemplum mismo, el ego epistolar ofrece su registro textual como una experiencia directa al lector, y así consigue transferir la auctoritas del discurso de Baso hacia su propio texto, condición sine qua non para dar perdurabilidad a la performance de Baso $^{53}$. Otra cuestión que incrementa la auctoritas de este registro textual es la exhibición por parte del ego epistolar del hecho de que Baso fue sometido a una dilatada pesquisa. Es decir, Baso es un exemplum «probado». La dificultad de distinguir un comportamiento ante la muerte verdaderamente virtuoso, internamente motivado, de una actitud ante ella que surja como resultado de presiones externas justifica el seguimiento minucioso que el ego epistolar ha hecho de Baso:

Fateor ergo ad hominem mihi carum ex pluribus me causis frequentius uenisse, ut scirem an illum totiens eundem inuenirem, numquid cum corporis uiribus minueretur animi uigor; qui sic crescebat illi quomodo manifestior notari solet agitatorum laetitia cum septimo spatio palmae appropinquat ${ }^{54}$.

Al presuponer que la entereza con que Baso sobrelleva su vejez y muerte próximas declinará con sus fuerzas, el ego epistolar anticipa la desconfianza del lector, quien podría sospechar que el comportamiento ejemplar de Baso podría sufrir un quiebre a medida que el fin se aproxima ${ }^{55}$. Lo que se destaca,

${ }^{53}$ Asimismo, puede considerarse que la jerarquía aquí establecida anticipa lo preconizado en la Ep. 52.8 con respecto a cuál ha de ser el criterio clave para elegir a alguien como modelo entre los presentes: eum elige adiutorem quem magis admireris cum uideris quam cum audieris.

${ }^{54}$ Sen., Ep. 30.13.

${ }^{55}$ La locuacidad y las abstracciones filosóficas previas a la muerte podían ser consideradas como una cobarde postergación. Cf., en este sentido, Tac., Hist. II 47, pasaje en el que se refiere la muerte de Otho: Plura de extremis loqui pars ignauiae est. Cf., asimismo, Sen., Ep. 26.6: mors de te pronuntiatura est. Ita dico: disputationes et litterata colloquia et ex praeceptis sapientium uerba collecta et eruditus sermo non ostendunt uerum robur animi; est enim oratio etiam timidissimis audax. Quid egeris tunc apparebit cum animam ages. 
en cambio, es la inmovilidad de Baso, que, al igual que Sócrates, se mantiene en todo momento igual a sí mismo ${ }^{56}$, haciendo frente a su muerte próxima con espíritu sereno (aequo animo, según lo preconizado en la Ep. 4.5) ${ }^{57}$. El valor de la compostura de Baso se ve incrementado notablemente entonces pues la vejez hace que su muerte se prolongue en el tiempo. Podemos pensar, además, que el texto propone a Baso como un nuevo tipo de héroe, que entrega a la posteridad no una hazaña ejemplar, sino su propia inacción, su ausencia de una respuesta desesperada, emocional, ante las dolorosas demandas de la enfermedad.

El cierre de la carta enfatiza que ha sido la materialidad de la escritura la que ha hecho posible registrar y dar perdurabilidad a la actitud asumida por Baso ante su muerte próxima, apuntando así a la ejemplaridad del propio texto, no sólo a del escritor ${ }^{58}$. En efecto, el ego epistolar concluye su texto con una broma que nos recuerda que estamos leyendo una carta: Sed uereri debeo ne tam longas epistulas peius quam mortem oderis. Itaque finem faciam: tu tamen mortem ut numquam timeas semper cogita. Vale (30.18). Es notable que la muerte efectiva de Baso, al igual que los detalles de su sufrimiento físico, se sustraigan completamente del registro que hace el ego epis$\operatorname{tolar}^{59}$, como si lo único que importara verdaderamente fuera el discurso que

${ }^{56} \mathrm{Cf}$. Viginti et septem annis pugnatum est; post finita arma triginta tyrannis noxae dedita est ciuitas, ex quibus plerique inimici erant. Nouissime damnatio est sub grauissimis nominibus impleta: obiecta est et religionum uiolatio et iunentutis corruptela, quam inmittere in deos, in patres, in rem publicam dictus est. Post haec carcer et uenenum. Haec usque eo animum Socratis non mouerant ut ne uultum quidem mouerint. $\langle O>$ illam mirabilem laudem et singularem! usque ad extremum nec hilariorem quisquam nec tristiorem Socraten uidit; aequalis fuit in tanta inaequalitate fortunae (Sen., Ep. 104.28).

${ }^{57} \mathrm{La}$ idea de que la proximidad de la muerte ofrece una prueba definitiva del carácter de un individuo se encuentra también en Lucr. III 55-58: quo magis in dubiis hominem spectare periclis / conuenit aduersisque in rebus noscere quid sit; / nam uerae uoces tum demum pectore ab imo/ eliciuntur et eripitur persona, manet res.

${ }^{58}$ Coincidimos con Edwards 2007, p. 145, en que «...the written form...inevitably raises questions of authorship. How far is the beautifully articulated death the creation of the dying subject - and how far is it the composition of the author who recounts it?».

${ }_{59}$ Esto resulta más llamativo si se considera que «Seneca's writing, both prose and verse, betrays a fascination with the physical details of bodily suffering...» (Edwards 1999, p. 264). Para un ejemplo de esta fascinación por los detalles, cf. Sen., Ep. 70.20: secessit ad exonerandum corpus - nullum aliud illi dabatur sine custode secretum; ibi lignum id quod ad emundanda obscena adhaerente spongia positum est totum in gulam farsit et interclusis faucibus spiritum elisit. 
éste pronuncia sobre su muerte próxima, su condición de texto viviente, capaz de eclipsar otros discursos leídos u oídos sobre el mismo tema ${ }^{60}$. Por otra parte, al subrayar la necesidad de cerrar la carta (finem faciam), el ego epistolar refuerza una vez más su paralelo con Baso, en la medida en que ambos están «terminando algo», aunque Baso lo hace oralmente y Séneca por escrito, con lo cual sugiere que la vida y la escritura son coextensivas e incluso intercambiables ${ }^{61}$.

\section{CONCLUSIÓN}

Si se concede que el ego epistolar se configura como exemplum a lo largo de esta epistula cabe que nos preguntemos cuál podría ser la ventaja de esta autopresentación. Una primera respuesta que sugerimos es que se trata de un procedimiento que resulta redituable para negociar la propia identidad y asegurarse un lugar en la memoria colectiva, hecho que se ve reforzado si se atiende a que Séneca refiere explícitamente su intención de publicar las Epistulae ${ }^{62}$. La cualidad de monumentum de la palabra escrita, con su capacidad de llegar a amplias audiencias a través del espacio y del tiempo, asegurando así el renombre del autor, proveía un vehículo muy conveniente para revisar, ajustar o corroborar la persona presentada en la vida pública ${ }^{63}$,

${ }^{60}$ Cf. Sen., Ep. 30.7: Haec ego scio et saepe dicta et saepe dicenda, sed neque cum legerem aeque mihi profuerunt neque cum audirem iis dicentibus qui negabant timenda a quorum metu aberant: hic uero plurimum apud me auctoritatis habuit, cum loqueretur de morte uicina.

${ }^{61}$ Cf. Sen., Ep. 58.37: Sed in longum exeo; est praeterea materia quae ducere diem possit: et quomodo finem imponere uitae poterit qui epistulae non potest? Vale ergo: quod libentius quam mortes meras lecturus es. Vale. Es habitual en Séneca utilizar las convenciones de la retórica epistolar, en este caso, una disculpa por una carta excesivamente larga, para dar pie a la reflexión filosófica. Cf., asimismo, Sen., Ep. 15.1: Mos antiquis fuit, usque ad meam seruatus aetatem, primis epistulae uerbis adicere 'si uales bene est, ego ualeo'. Recte nos dicimus 'si philosopharis, bene est'. Valere enim hoc demum est. Sine hoc aeger est animus; corpus quoque, etiam si magnas habet uires, non aliter quam furiosi aut frenetici ualidum est. Para este tema, cf. Cugusi 1983, pp. 28-29.

${ }^{62}$ Cf. Sen., Ep. 21.4. A pesar de que sería sumamente útil para nuestra hipótesis contar con más detalles sobre los modos de circulación del epistolario senecano, todo lo que sabemos sobre este tema se encuentra en el terreno de la especulación (Ker 2009, p. 149).

${ }^{63}$ Cf. Roller 2011, p. 214. Los estudios del concepto de self en la obra de Séneca coinciden en señalar el hecho de que la concepción subjetivo-individualista heredada de Descartes no puede serle aplicada, pues se trata de un ego que se revela a sí mismo en el modo en que 
cuestión central si consideramos que el epistolario fue escrito en los años finales de la vida de su autor, luego de que éste se retirara definitivamente de la escena política. Puede pensarse, incluso, que una característica central del exemplum, su carácter extraíble y, por lo tanto, «citable» ${ }^{64}$, podía contribuir a la propagación de la imagen que el ego epistolar deseaba forjar de sí mismo: una imagen de sí fundamentalmente espiritual y libresca, que dejaba por completo en la sombra a su persona política.

Esperamos entonces que nuestro análisis haya mostrado que, a pesar de que el ego epistolar no se pone en esta carta en primer plano como exemplum $^{65}$, su propio comportamiento, tal como es aquí presentado, es tan ejemplar como el de Baso. En efecto, mientras que Baso se instruye sobre cómo morir bien dedicándose a mantener conversaciones filosóficas con sus amigos sobre la necesidad de no temer a la muerte, el ego epistolar se presenta también como alguien que está aprendiendo a morir bien visitando y conversando con Baso, describiendo estas visitas y reflexionando sobre ellas. Así, mientras que Baso spectator ha estado contemplando su propia muerte como si fuese ajena, el ego epistolar ha estado observando esta muerte modélica como si fuera un simulacro o ensayo de su propio y esperado fin ${ }^{66}$.

actúa y en lo que comunica, es decir, un yo «performativo». Según Long 2009, p. 24: «This performative and hardly interior self links and unifies the author of the Letters and other prose works...to the author of the tragedies». Vale aclarar que esta característica no es privativa de Séneca sino que forma parte del papel que juega la mirada en la constitución de la identidad en Roma. Según Dupont 1992, p. 25, «Rome est une civilisation où l'identité morale n'est pas l'effet d'une conscience intérieure: cette identité, le Romain la reçoit du regard que la collectivité porte sur lui. Le Romain n'a d'autre image de soi que celle que lui renvoie le regard des autres». Para este tema, cf. Bartsch 2006.

${ }^{64}$ Recordemos que el sustantivo exemplum deriva del verbo eximo «to take out, extract» (cf. $O L D$, s. u.1).

${ }^{65}$ Podemos suponer que su evidente fracaso como consejero áulico de Nerón debilitaba sus credenciales para actuar como magister y presentarse a sí mismo como exemplum de manera directa.

${ }^{66}$ El círculo se cierra si consideramos el testimonio de Tácito sobre el modo ejemplar en que Séneca enfrentó su propia muerte (Tac., Ann. XV 60-64), si bien no podemos descartar cierta circularidad en este testimonio, dado que la propia obra de Séneca es el intertexto más evidente de la narración de Tácito. En todo caso, lo que sí es evidente en la narración de esta muerte «filosófica» es la fuerza prescriptiva del exemplum que los textos de Séneca han contribuido a forjar. 


\section{BIBLIOGRAFÍA}

Armisen-Marchetti, M. 1989: Sapientiae facies. Étude sur les images de Sénèque, París.

Barton, C. A. 1993: The Sorrows of the Ancient Romans: The Gladiator and the Monster, Princeton.

Bartsch, S. 2006: The Mirror of the Self. Sexuality, Self-Knowledge and the Gaze in the Early Roman Empire, Chicago.

Brown, R. D. 1992: «Senecan Drama and Stoic Cosmology», Ancient Philosophy 12 (2), pp. 479-483.

Cancik, H. 1967: Untersuchungen zu Senecas Epistulae morales, Hildesheim.

Chaplin, J. 2000: Livy's Exemplary History, Oxford.

Coffta, D. J. 2002: The influence of Callimachean aesthetics on the Satires and Odes of Horace. Studies in Classics 19, Lewiston-Queenston-Lampeter.

Cugusi, P. 1983: Evoluzione e forme dell'epistolografia latina nella tarda reppublica e nei primi due secoli dell'impero, con cenni sull'epistolografia preciceroniana, Roma.

Dupont, F. 1992: «Des images qui font parler», Résistances de l'image, T.I.G.R.E. (Texte et image, Groupe de Recherche à l' École Normale Supérieure), París, pp. 23-31.

Edwards, C. 1997: «Self-Scrutiny and Self- Transformation in Seneca's Letters», G\&R 44, pp. 23-38.

Edwards, C. 1999: «The suffering body: Philosophy and Pain in Seneca's Letters», en Porter, J. I. (ed.), Constructions of the Classical Body, Ann Arbor, pp. 252-268.

Edwards, C. 2007: Death in Ancient Rome, New Haven-Londres.

Gill, C. 1973: «The Death of Socrates», CQ 23, pp. 224-228.

Graver, M. R. 1996: Therapeutic Reading and Seneca's Moral Epistles, PhD Dissertation, Brown University.

Graver, M. R. 2016: «The Emotional Intelligence of Epicureans. Doctrinalism and Adaptation in Seneca's Epistles», en Williams, G. D. y Volk, K. (eds.), Roman Reflections: Studies in Latin Philosophy, Nueva York, pp. 192-210.

Griffin, M. T. 1992 [1976]: Seneca: A Philosopher in Politics, Oxford.

Habinek, T. 1982: «Seneca's circles: Ep. 12.6-9», CA 1, pp. 66-69.

Hill, T. D. 2004: Ambitiosa Mors. Suicide and Self in Roman Thought and Literature, Nueva York-Londres.

Inwood, B. 2009: «Seneca and self assertion», en Bartsch, S. y Wray, D. (eds.), Seneca and the Self, Cambridge, pp. 39-64.

Ker, J. 2009: The Deaths of Seneca, Oxford.

La Penna, A. 1994: «Un' altra eco di Lucrezio in Seneca? (con qualche riflessione sulla tradizione indiretta)», Maia 46, pp. 319-322. 
Lana, I. 1991: «Le "Lettere a Lucilio" nella letteratura epistolare», en Grimal, P. (ed.), Sénèque et la prose latine, Vandoeuvres-Génova, pp. 253-311.

Leeman, A. D. 1953: «Seneca's Plans for a Work "Moralis philosophia” and Their Influence on His Later Epistles», Mnemosyne 6, pp. 307- 313.

Leeman, A. D. 1966: «Seneca's Phaedra as a Stoic tragedy», en Bremer, J. M., Radt, S. L. y Ruijgh, G. J. (eds.), Miscellanea Tragica in Honorem J. C. Kamerbeek, Ámsterdam, pp. 199-212.

Long, A. A. 2009: «Seneca on the self: why now?», en Bartsch, S. y Wray, D. (eds.), Seneca and the Self, Cambridge, pp. 20-36.

Malherbe, A. J. (ed.) 1988: Ancient Epistolary Theorists, Atlanta.

Mann, W. R. 2006: «Learning How to Die: Seneca's Use of Aeneid 4.653 at Epistulae Morales 12.9», en Volk, K. y Williams, G. D. (eds.), Seeing Seneca Whole Perspectives on Philosophy and Politics, Leiden-Boston, pp. 103-22.

Marti, B. M. 1945: «Seneca's tragedies. A new interpretation», TAPhA 76, pp. 216-245.

Maurach, G. 1970: Der Bau von Senecas Epistulae Morales, Heidelberg.

Mayer, R. G. 2008: «Roman Historical Exempla in Seneca», en Fitch, J. (ed.), Seneca, Oxford, pp. 299-315.

Mazzoli, G. 1970: Seneca e la poesia, Milán.

Mazzoli, G. 1989: «L' Epistulae morales ad Lucilium di Seneca: Valore letterario e filosofico», ANRW 36 (2), pp. 1823-1877.

Muñoz Martín, N. 1985: Teoría epistolar y concepción de la carta en Roma, Granada.

Nussbaum, M. C. 1994: The Therapy of Desire. Theory and Practice in Hellenistic Ethics, Princeton.

Pociña Pérez, A. 1976: «Finalidad político-didáctica de las tragedias de Seneca», Emerita 44, pp. 279-301.

Pociña Pérez, A. 1978: «Las tragedias latinas de tesis», Emerita 46, pp. 1-11.

Pratt, N. T. 1948: «The Stoic base of Senecan drama», TAPhA 79, pp. 1-11.

Reinhardt, T. 2016: «To See and to Be Seen: On Vision and Perception in Lucretius and Cicero», en Williams, G. D. y Volk, K. (eds.), Roman Reflections: Studies in Latin Philosophy, Nueva York, pp. 63-90.

Reynolds, L. D. (ed.) 1965: L. Annaei Senecae ad Lucilium Epistulae Morales, Oxford.

Roller, M. 2001: Constructing Autocracy. Aristocrats and Emperors in Julio-Claudian Rome, Princeton.

Roller, M. 2004: «Exemplarity in Roman Culture: The Cases of Horatius Cocles and Cloelia», CPh 99, pp. 1-56.

Roller, M. 2011: «To whom am I speaking? The changing venues of competitive eloquence in the early empire», en Blösel, W. y Hölkeskamp, K. J. (eds.), Von der militia equestris zur militia urbana: Prominenzrollen und Karrierefelder im antiken Rom, Stuttgart, pp. 197-221. 
Roller, M. 2015: «Between unique and typical: Senecan exempla in a list», en Lowrie, M. y Lüdemann, S. (eds.), Exemplarity and Singularity. Thinking through Particulars in Philosophy, Literature, and Law, Londres y Nueva York, pp. 81-95.

Roller, M. 2016: «Praecept(or) and Example in Seneca», en Williams, G. D. y Volk, K. (eds.), Roman Reflections: Studies in Latin Philosophy, Nueva York, pp. 129-156.

Russell, D. A. 1974: «Letters to Lucilius», en Costa, C. D. N. (ed.), Seneca, Boston, pp. 70-95.

Schiesaro, A. 2009: «Exemplarity: Between Practice and Text», en Maes, Y., Papy, J. y Verbaal, W. (eds.), Latinitas Perennis. Vol. II: Appropriation and Latin Literature, Leiden-Boston, pp. 41-64.

Schiesaro, A. 2015: "Seneca and Epicurus: The Allure of the Other», en Bartsch, S. y Schiesaro, A. (eds.), The Cambridge Companion to Seneca, Cambridge, pp. 239-251.

Schottlaender, R. 1955: «Epikureisches bei Seneca: Ein Ringen um den Sinn von Freude und Freundschaft», Philologus 99, pp. 133-148.

Setaioli, A. 1991: «Seneca e gli arcaici», en Setaioli, A. (ed.), Seneca e la cultura: Atti del Convegno di Perugia, 9-10 novembre 1989, Nápoles, pp. 33-45.

Solimano, G. 1991: La prepotenza dell'occhio. Riflessioni sull'opera di Seneca, Génova.

Syme, R. 1958: Tacitus, Oxford.

Walter, U. 2004: Memoria und res publica: zur Geschichtskultur im republikanischen Rom, Frankfurt am Main.

Wilcox, A. 2012: The Gift of Correspondence in Classical Rome. Friendship in Cicero's Ad Familiares and Seneca's Moral Epistles, Madison.

Wildberger, J. 2014: «The Epicurus Trope and the Construction of a "Letter Writer"», en Wildberger, J. y Colish, M. L. (eds.), Seneca Philosophus, Berlín-Boston, pp. 431-465.

Wilson, M. 1987: «Seneca’s Epistles to Lucilius: A Revaluation», en Boyle, A. J. (ed.), The Imperial Muse, Berwick, Victoria, pp.102-121.

Wilson, M. 2001: «Seneca’s Epistles Reclassified», en Harrison, S. J. (ed.), Texts, Ideas, and the Classics, Oxford, pp. 164-188.

Fecha de recepción de la primera versión del artículo: 30/05/2016

Fecha de aceptación: 16/10/2016

Fecha de recepción de la versión definitiva: 18/10/2016 